\title{
SHOCK FORMATION IN A MULTIDIMENSIONAL VISCOELASTIC DIFFUSIVE SYSTEM*
}

\author{
DONALD S. COHEN ${ }^{\dagger}$, ANDREW B. WHITE, JR. ${ }^{\ddagger}$, AND THOMAS P. WITELSKI ${ }^{\S}$ \\ This paper is dedicated to Joseph B. Keller on the occasion of his 70 th birthday.
}

\begin{abstract}
We examine a model for non-Fickian "sorption overshoot" behavior in diffusive polymer-penetrant systems. The equations of motion proposed by Cohen and White [SIAM J. Appl. Math., 51 (1991), pp. 472-483] are solved for two-dimensional problems using matched asymptotic expansions. The phenomenon of shock formation predicted by the model is examined and contrasted with similar behavior in classical reaction-diffusion systems. Mass uptake curves produced by the model are examined and shown to compare favorably with experimental observations.
\end{abstract}

Key words. viscoelastic diffusion, shocks, reaction-diffusion

AMS subject classifications. 35K57, 35K60, 35K22, 35C20, $73 \mathrm{~F} 15$

1. Introduction. Polymeric materials have many distinguishing properties that differentiate them from classical materials. These properties lead to nontraditional effects that are being exploited to yield innovative new technologies. Widespread uses of polymers in engineering, scientific, and commercial applications necessitate development of theories that will accurately describe their behavior. Numerous experiments have produced a broad range of nonlinear phenomena generically called "non-Fickian behavior" which cannot be explained using the classical diffusion model [4], [22]. A model proposed to describe the "sorption overshoot" behavior will be examined here. This paper extends the model presented by Cohen and White [3], [4] to two-dimensional problems and analyzes aspects of the shock formation and mass-uptake characteristics of the model.

In $\S 2$ we derive the model for the viscoelastic diffusive system. In $\S 3$ the initialboundary value problem is formulated and its time-independent solution is analyzed. Section 4 presents a solution of the problem using the method of matched asymptotic expansions. In $\S 5$ we compare our system to classical reaction-diffusion systems and examine the nature of shock formation. Finally, in $\S 6$ we conclude with an analysis of the mass-uptake curves produced by the model.

2. The model. Classical continuum models for diffusion in materials are described by an equation,

$$
\frac{\partial c}{\partial T}=-\nabla \cdot \mathbf{J}
$$

* Received by the editors June 26, 1993; accepted for publication (in revised form) May 27, 1994.

$\dagger$ Department of Applied Mathematics, 217-50, California Institute of Technology, Pasadena, California 91125. The research of this author was supported by United States Army Research Office (Durham) contract DAAL03-89-K-0014, National Science Foundation grant DMS-9024963, Air Force Office of Scientific Research grant AFOSR-91-0045, and Department of Energy grant W-7405-ENG36 and performed at the Center for Nonlinear Studies at Los Alamos.

$\ddagger$ Advanced Computer Laboratory, Los Alamos National Laboratory, Los Alamos, New Mexico 87545. The research of this author was supported by United States Department of Energy contract W-7405-ENG-36.

$\S$ Department of Applied Mathematics, 217-50, California Institute of Technology, Pasadena, California 91125 . The research of this author was supported by a National Science Foundation graduate fellowship, National Science Foundation grant DMS-9024963, and Air Force Office of Scientific Research grant AFOSR-91-0045. 
for the conservation of mass, where $c(X, Y, T)$ is the local concentration and $\mathbf{J}$ is the concentration flux vector. Fick's law,

$$
\mathbf{J}=-\underline{\mathbf{D}}(c) \nabla c,
$$

postulates that the flux is proportional to the negative gradient of concentration, where $\underline{\mathbf{D}}(c)$ is a positive-definite diffusion-coefficient tensor. Use of flux (2.2) in (2.1) leads to the standard Fickian diffusion equation

$$
\frac{\partial c}{\partial T}=\nabla \cdot(\underline{\mathbf{D}}(c) \nabla c)
$$

If $\underline{\mathbf{D}}(c)$ is constant and isotropic, $\underline{\mathbf{D}}(c)=D \underline{\mathbf{1}}$, then (2.3) reduces to the classical heat equation

$$
\frac{\partial c}{\partial T}=D \nabla^{2} c
$$

Various experimentally observed behaviors in polymer diffusion cannot be explained using (2.3). One class of absorption experiments consists of measuring massuptake and concentration of a penetrant in a thin polymer film substrate. The mass of penetrant absorbed by the polymer film increases sharply with time until some point when it begins to decrease gradually to a steady-state value. This behavior is called "sorption overshoot." Thomas and Windle [21] observed viscoelastic relaxation processes as the large polymer molecules rearrange (bend, twist, and shift position) during the diffusion process and suggest that the flux contain a term reflecting this effect. Vrentas, Duda, and Hou similarly suggest that this kind of "sluggish" mechanical relaxation is responsible for overshoot [22]. Once the importance of a relaxation mechanism is recognized, it is clear that the classical diffusion models, all of which ignore this mechanism, are incapable of describing the observed phenomena.

Cohen and White suggest a flux containing a contribution from viscoelastic stress in the polymer film. Such a form is

$$
\mathbf{J}=-(D \nabla c+E \nabla \cdot \underline{\sigma})
$$

where $E$ is a positive stress-diffusion coefficient. If we assume that the diffusion of the penetrant in the polymer substrate does not cause any deformations or deviatoric stresses (or if deviatoric stresses are negligible) then we may take the stress to be isotropic, $\underline{\sigma}=\sigma \underline{1}$, and the flux becomes

$$
\mathbf{J}=-(D \nabla c+E \nabla \sigma)
$$

Cohen and White propose a viscoelastic relaxation equation for $\sigma$,

$$
\frac{\partial \sigma}{\partial T}+\beta(c) \sigma=\mu c
$$

The properties of the polymer material are specified by giving the constant $\mu$ and the function $1 / \beta(c)$, the mechanical relaxation time for the polymer substrate as a function of the penetrant concentration. For low penetrant concentrations the polymer is in a relatively inflexible "glassy" state with a long relaxation time $1 / \beta_{G}$. For higher penetrant concentrations the polymer takes on a more responsive "rubbery" state 
with shorter relaxation time $1 / \beta_{R}, \beta_{R}>\beta_{G}$. This change in state occurs at a rubberglass transition concentration $c_{R G}$ over a range of concentrations of order $\Delta$ and can be modeled using

$$
\beta(c)=\frac{1}{2}\left(\beta_{R}+\beta_{G}\right)+\frac{1}{2}\left(\beta_{R}-\beta_{G}\right) \tanh \left(\frac{c-c_{R G}}{\Delta}\right)
$$

(see Fig. 1). If we assume that we are starting from a dry polymer with no viscoelastic pressure $\sigma(\mathbf{X})=0$ at $T=0$, then we can integrate (2.7) to obtain

$$
\sigma(\mathbf{X}, T)=\mu \int_{0}^{T} e^{-\int_{\tau}^{T} \beta(c(\mathbf{X}, s)) d s} c(X, Y, \tau) d \tau .
$$

Combining (2.1), (2.6), and (2.9), we obtain the single equation

$$
\frac{\partial c}{\partial T}=\nabla \cdot\left(D \nabla c+\mu E \nabla \int_{0}^{T} e^{-\int_{\tau}^{T} \beta(c) d s} c d \tau\right)
$$

in which a hereditary (or memory) integral [3], [4] is explicitly displayed. While the form (2.10) is useful for many purposes, for our analysis we will consider the coupled system (2.1), (2.6), (2.7).

An alternate and perhaps more general derivation of the model is given by energy considerations in the system. For polymer-penetrant systems near the glassrubber transition where molecular diffusion and relaxation are the dominant physical mechanisms, the integro-differential model (2.10) is particularly appealing even when significant deformation and shear occurs. To derive this model under these broader conditions we can proceed as follows: if $\mathcal{U}$ is, in some sense, the internal energy density of the system, then a chemical potential $\mu_{0}$ for diffusion is defined by

$$
\mu_{0}=\frac{\delta \mathcal{U}}{\delta c}
$$

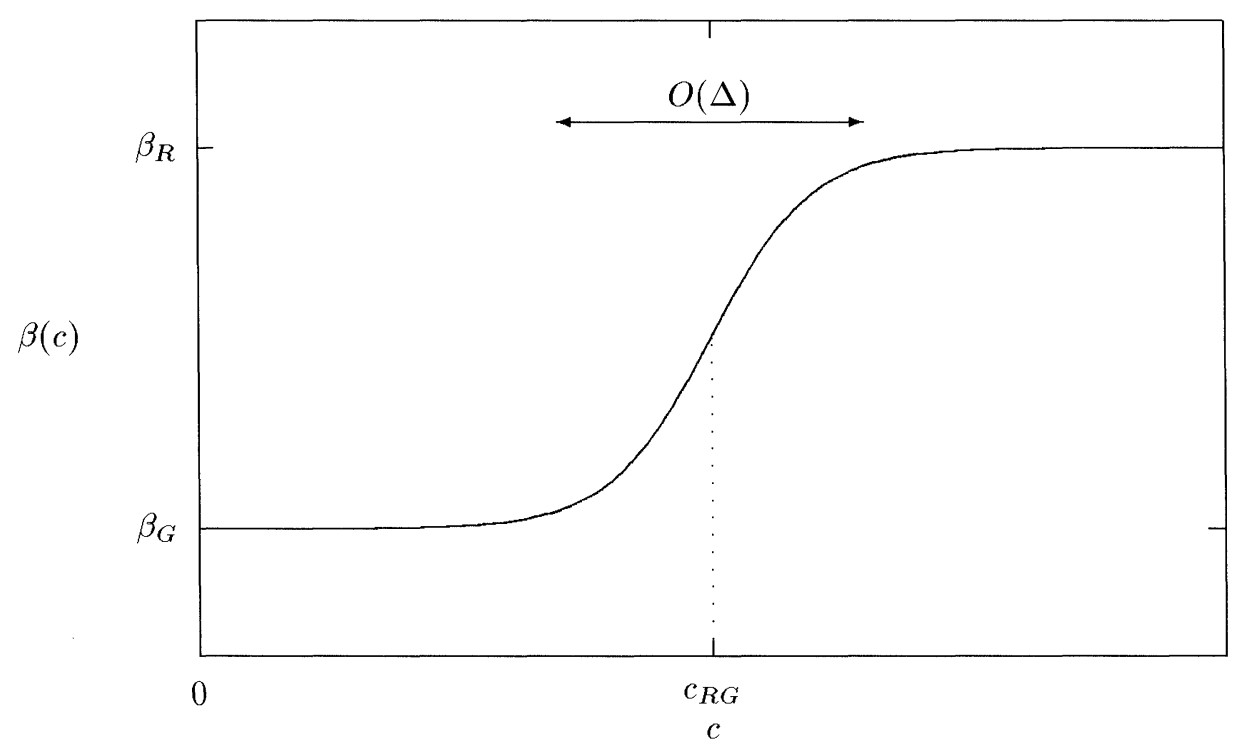

FIG. 1. The inverse relaxation time. 
The diffusive concentration flux is proportional to the gradient of the potential $\mu_{0}$ so that

$$
\begin{aligned}
\mathbf{J} & =-k(c) \nabla \mu_{0} \\
& =-k(c) \mu_{0}^{\prime}(c) \nabla c \\
& =-D(c) \nabla c,
\end{aligned}
$$

where $D(c)=k(c) \mu_{0}^{\prime}(c)$.

This is Fick's law, (2.2), where the diffusivity $D(c)$ is now given by $k(c) \mu_{0}^{\prime}(c)$. Thus, the phenomenological formulation (2.2) becomes a consequence of the more basic postulate that the diffusive chemical potential $\mu_{0}$ is state dependent only.

In addition to diffusive transport, the various phenomena which contribute to the relaxation process will increase the system's internal energy and also its chemical potential. Thus, to the diffusive potential $\mu_{0}$ we add a potential $\mu_{\tau}$ due to relaxation. Furthermore, we argue that $\mu_{\tau}$ must have the form typical of all relaxation processes [19], namely a hereditary integral. Therefore, the total chemical potential $\mu$ is given by

$$
\mu=\mu_{0}+\mu_{\tau}
$$

where

$$
\mu_{\tau}=\int_{-\infty}^{T} e^{-\int_{t}^{T} \beta(c(\mathbf{X}, s)) d s} f\left(c(\mathbf{X}, t), \frac{\partial c}{\partial T}(\mathbf{X}, t)\right) d t
$$

The function $f\left(c, \frac{\partial c}{\partial T}\right)$ represents the dependence of relaxation on the concentration field and its rates of change [7], [20]. In (2.10), $f$ is taken to be simply proportional to the concentration.

The total concentration flux is now the gradient of the potential $\mu$ so that

$$
\begin{aligned}
\mathbf{J} & =-k(c) \nabla\left(\mu_{0}+\mu_{\tau}\right) \\
& =-D(c) \nabla c-E(c) \nabla \int_{-\infty}^{T} e^{-\int_{t}^{T} \beta(c(\mathbf{X}, s)) d s} f\left(c(\mathbf{X}, t), \frac{\partial c}{\partial T}(\mathbf{X}, t)\right) d t
\end{aligned}
$$

where $D(c)=k(c) \mu_{0}^{\prime}(c)$ and $E(c)=k(c)$.

Finally, the basic equation of conservation of mass (2.1) becomes

$$
\frac{\partial c}{\partial T}=\nabla \cdot\left[D(c) \nabla c+E(c) \nabla \int_{-\infty}^{T} e^{-\int_{t}^{T} \beta(c(\mathbf{X}, s)) d s} f\left(c(\mathbf{X}, t), \frac{\partial c}{\partial T}(\mathbf{X}, t)\right) d t\right]
$$

Equation (2.16), and its obvious further generalizations, is now meant to replace the classical equation (2.4) for use in situations where various relaxation mechanisms are of comparable physical importance to purely diffusive transport. Mechanical and/or chemical relaxation mechanisms are accounted for without the need to specify the form of the accompanying strains. Successful use of the model has already been achieved for Case II diffusion [8]-[14].

In using the specific form (2.16) for studies of Case II transport, we have found it convenient to replace (2.16) by a pair of coupled differential equations, as was considered earlier in this section $(2.1),(2.6),(2.7)$. These equations subject to various appropriate initial and boundary conditions at fixed and moving boundaries constitute well-posed formulations for a wide spectrum of problems. 
It should be noted that standard equilibrium boundary conditions (Dirichlet, Neumann, or a linear combination of the two) do not accurately model physical phenomena in many cases. It is necessary to account for the nonequilibrium contribution to the chemical potential because of relaxation effects. These considerations yield more complicated time-dependent boundary conditions and are discussed more fully in [15] and [16]. In [15] there is also a discussion of the still more complicated conditions to be imposed at moving boundaries.

3. The problem. We will consider a two-dimensional initial-boundary value problem on a finite domain, $0<X<L, 0<Y<L_{Y}$, corresponding to a cross-section of a polymer film in a sorption experiment. For simplicity, the diffusion coefficients $\underline{\mathbf{D}}, \underline{\mathbf{E}}$ will be taken to be constant and isotropic and the substrate will be initially dry, "unstressed" polymer (3.1c). Concentration on the left and right edges of the domain are given (3.1d), and the top and bottom edges of the domain have insulated (no-flux) boundary conditions (3.1e).

$$
\begin{gathered}
\frac{\partial c}{\partial T}=D \nabla^{2} c+E \nabla^{2} \sigma \\
\frac{\partial \sigma}{\partial T}+\beta(c) \sigma=\mu c \\
c(X, Y, 0)=0, \quad \sigma(X, Y, 0)=0, \\
c(0, Y, T)=c^{0}(Y), \quad c(L, Y, T)=c^{1}(Y), \\
\left.\mathbf{J} \cdot \hat{\mathbf{n}}\right|_{Y=0, L_{Y}}=0 \quad \text { or } \quad D \frac{\partial c}{\partial Y}+\left.E \frac{\partial \sigma}{\partial Y}\right|_{Y=0, L_{Y}}=0 .
\end{gathered}
$$

We introduce dimensionless variables using the characteristic scalings given below

$$
\begin{gathered}
c=C u, \quad \sigma=\frac{D C}{E} s, \\
X=L x, \quad Y=L y, \quad T=\frac{D}{\mu E} t, \\
\epsilon=\frac{\mu E L^{2}}{D^{2}}, \quad \lambda=\frac{D \beta_{R}}{\mu E}, \\
\frac{\beta(c)}{\beta_{R}}=b(u)=\frac{1+\omega}{2}+\frac{1-\omega}{2} \tanh \left(\frac{u-u_{R G}}{\delta}\right), \\
\omega=\frac{\beta_{G}}{\beta_{R}}<1, \quad \Delta=C \delta, \quad c_{R G}=C u_{R G}, \quad l=\frac{L_{Y}}{L} .
\end{gathered}
$$

Then, on $0<x<1,0<y<l$, we obtain

$$
\epsilon \frac{\partial u}{\partial t}=\nabla^{2} u+\nabla^{2} s
$$




$$
\begin{gathered}
\frac{\partial s}{\partial t}+\lambda b(u) s=u \\
u(x, y, 0)=0, \quad s(x, y, 0)=0, \\
u(0, y, t)=u^{0}(y), \quad u(1, y, t)=u^{1}(y), \\
\left.\left(\frac{\partial u}{\partial y}+\frac{\partial s}{\partial y}\right)\right|_{y=0, l}=0 .
\end{gathered}
$$

It will be shown that (3.3a-e) approaches a steady-state solution as $t \rightarrow \infty$. Determination of this solution requires an examination of the full initial-boundary problem. However, a preliminary consideration of the time-independent solutions of $(3.3 \mathrm{a}-\mathrm{e})$ provides some insights to the problem. A time-independent solution, $\bar{u}(x, y), \bar{s}(x, y)$, of $(3.3 \mathrm{a}-\mathrm{e})$ must satisfy

$$
\begin{gathered}
\nabla^{2}(\bar{u}+\bar{s})=0 \\
\lambda b(\bar{u}) \bar{s}=\bar{u} \\
\bar{u}(0, y)=u^{0}(y), \quad \bar{u}(1, y)=u^{1}(y) \\
\left.\left(\frac{\partial \bar{u}}{\partial y}+\frac{\partial \bar{s}}{\partial y}\right)\right|_{y=0, l}=0 .
\end{gathered}
$$

Solving (3.4b) for $\bar{s}$ yields

$$
\bar{s}=\frac{\bar{u}}{\lambda b(\bar{u})} .
$$

We recast $(3.4 \mathrm{a}-\mathrm{d})$ in a more convenient form in terms of the variable $\bar{w}$ given by

$$
\bar{w}(x, y)=\bar{u}(x, y)+\bar{s}(x, y) .
$$

It is also useful to define the function $W(u)$ as

$$
W(u) \equiv u+\frac{u}{\lambda b(u)}
$$

Using (3.6) and (3.7), (3.4a,c,d) can be written for $\bar{w}$ as

$$
\begin{gathered}
\nabla^{2} \bar{w}=0 \\
\bar{w}(0, y)=w^{0}(y) \equiv W\left(u^{0}(y)\right), \quad \bar{w}(1, y)=w^{1}(y) \equiv W\left(u^{1}(y)\right), \\
\left.\frac{\partial \bar{w}}{\partial y}\right|_{y=0, l}=0 .
\end{gathered}
$$

Problem $(3.8 \mathrm{a}-\mathrm{c})$ is a Laplace problem for $w$ with Dirichlet-Neumann boundary conditions. From (3.5)-(3.7) we see that $\bar{u}$ is related to $\bar{w}$ through

$$
\bar{w}(x, y)=W(\bar{u}(x, y)) .
$$


It is necessary to invert (3.9) at each point $(x, y)$ in the domain to find $u$ in terms of $w$. Since the inverse function $\bar{u}=W^{-1}(\bar{w})$ is triple-valued in part of its range for sufficiently small $\delta$ in $(3.2 \mathrm{~d})$ (see Fig. 2), the solution $\bar{u}(x, y)$ will generally be a triple-valued surface (see the triple-valued surface plotted in Fig. 3). This multivalued time-independent solution cannot be the steady-state solution of $(3.3 \mathrm{a}-\mathrm{e})$. This leads us to suspect that the actual steady state will contain a shock-like structure connecting single-valued branches of $\bar{u}$ (see Fig. 4); this will be shown to be the case in the following sections.

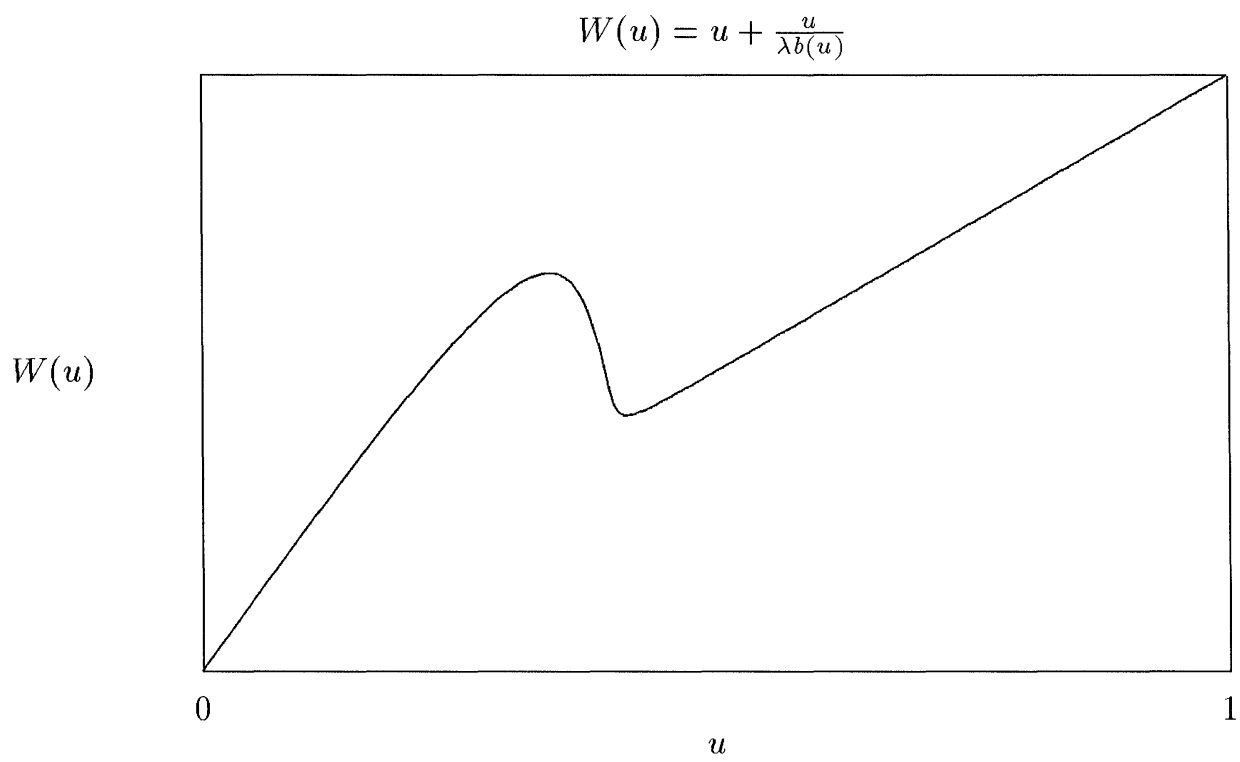

FIG. 2. The function $W(u)$.

4. Asymptotic solution. We will derive an approximate asymptotic solution in the limit of large diffusion for which $\epsilon \ll 1$. We construct a solution for all times using the method of matched asymptotic expansions.

4.1. Initial layer. For $\epsilon \ll 1$, we expect there to exist a short initial period where the solution evolves under the influence of the initial conditions. By rescaling time as

$$
\tilde{t}=t / \epsilon,
$$

the system $(3.3 \mathrm{a}, \mathrm{b})$ becomes

$$
\begin{aligned}
& \frac{\partial u}{\partial \tilde{t}}=\nabla^{2} u+\nabla^{2} s, \\
& \frac{\partial s}{\partial \tilde{t}}=\epsilon(u-\lambda b(u)) .
\end{aligned}
$$

In these variables,

$$
u(x, y, \tilde{t})=\sum_{n=0}^{\infty} \epsilon^{n} U_{n}(x, y, \tilde{t})=U_{0}(x, y, \tilde{t})+\epsilon U_{1}(x, y, \tilde{t})+\cdots
$$




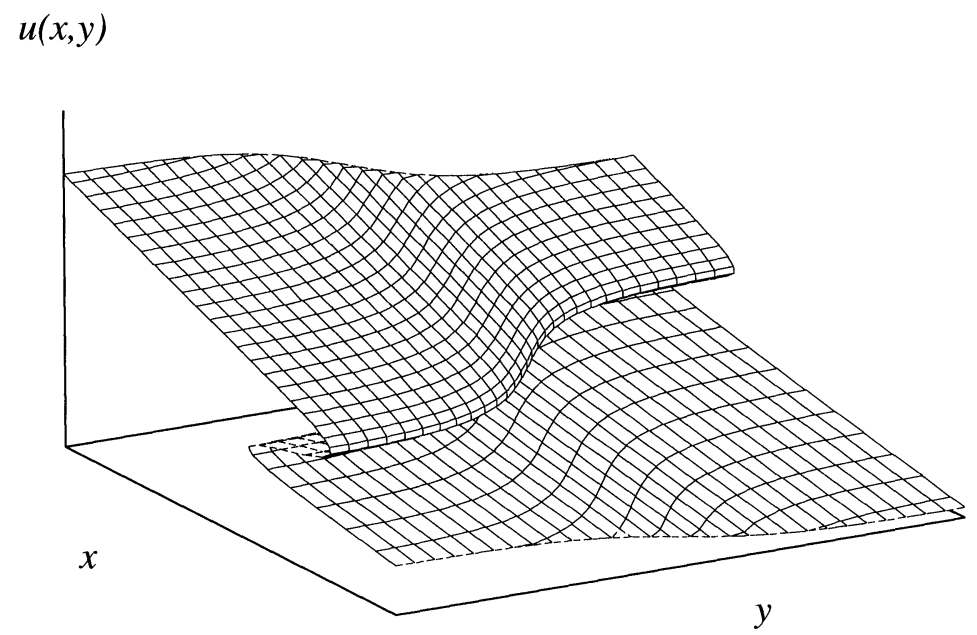

FIG. 3. The triple-valued surface $\bar{u}(x, y)$.

$$
s(x, y, \tilde{t})=\sum_{n=0}^{\infty} \epsilon^{n} S_{n}(x, y, \tilde{t})=S_{0}(x, y, \tilde{t})+\epsilon S_{1}(x, y, \tilde{t})+\cdots
$$

Substituting these expansions into $(4.2 \mathrm{a}, \mathrm{b})$ and retaining the leading order terms yields

$$
\begin{gathered}
\frac{\partial U_{0}}{\partial \tilde{t}}=\nabla^{2} U_{0}+\nabla^{2} S_{0} \\
\frac{\partial S_{0}}{\partial \tilde{t}}=0 .
\end{gathered}
$$

Applying the initial conditions implies that

$$
\begin{gathered}
S_{0}=0, \\
\frac{\partial U_{0}}{\partial \tilde{t}}=\nabla^{2} U_{0} .
\end{gathered}
$$

Therefore, to leading order, the concentration is purely diffusive in the initial layer. To match this initial layer to an outer solution that will hold for large finite time, let $\tilde{t} \rightarrow \infty$, so that the initial layer solution approaches the steady-state solution $\underline{U_{0}}(x, y)$

$$
\nabla^{2} \underline{U_{0}}=0
$$

$$
\underline{U_{0}}(0, y)=u^{0}(y), \quad \underline{U_{0}}(1, y)=u^{1}(y)
$$




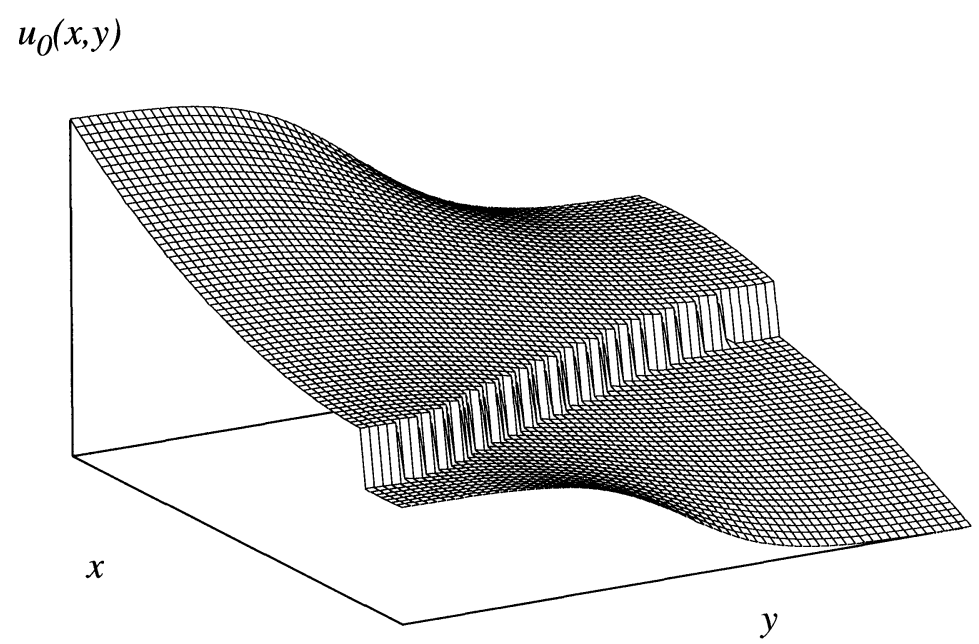

FIG. 4. The single-valued steady-state solution containing a shock.

$$
\left.\frac{\partial \underline{U_{0}}}{\partial y}\right|_{y=0, l}=0 .
$$

This steady state will be the initial condition for the outer solution.

4.2. Outer solution. Away from the initial layer, the outer solution can be written as

$$
\begin{aligned}
& u(x, y, t)=\sum_{n=0}^{\infty} \epsilon^{n} u_{n}(x, y, t)=u_{0}(x, y, t)+\epsilon u_{1}(x, y, t)+\cdots \\
& s(x, y, t)=\sum_{n=0}^{\infty} \epsilon^{n} s_{n}(x, y, t)=s_{0}(x, y, t)+\epsilon s_{1}(x, y, t)+\cdots
\end{aligned}
$$

Substituting these expansions into $(3.3 \mathrm{a}, \mathrm{b})$ and retaining leading order terms yields

$$
\begin{gathered}
\nabla^{2}\left(u_{0}+s_{0}\right)=0 \\
\frac{\partial s_{0}}{\partial t}+\lambda b\left(u_{0}\right) s_{0}=u_{0} .
\end{gathered}
$$

From the exact problem $(3.3 \mathrm{a}-\mathrm{e})$ it is possible to determine the boundary values of $s$ at $x=0,1$. To do so we note that $(3.3 \mathrm{~b})$ is purely evolutionary and has no explicit spatial dependence. Therefore it is uncoupled from (3.3a) and may be solved explicitly at $x=0,1$ where the boundary values of $u$ are given for all time. Thus, at 
$x=0,(3.3 \mathrm{~b})$ becomes

$$
\frac{d s}{d t}+\lambda b\left(u^{0}(y)\right) s=u^{0}(y)
$$

the solution of which is

$$
s(0, y, t)=s^{0}(y, t)=\frac{u^{0}(y)}{\lambda b\left(u^{0}(y)\right)}\left(1-e^{-\lambda b\left(u^{0}(y)\right) t}\right) .
$$

Similarly, at $x=1$, we obtain

$$
s(1, y, t)=s^{1}(y, t)=\frac{u^{1}(y)}{\lambda b\left(u^{1}(y)\right)}\left(1-e^{-\lambda b\left(u^{1}(y)\right) t}\right) .
$$

Note that these solutions satisfy the initial conditions at $t=0$. If the boundary conditions on $u$ at $x=0,1$ were time-dependent, then $s^{0}, s^{1}$ could be generally determined using numerical integration. This allows us to easily extend our analysis to cover problems with time-dependent nonequilibrium boundary conditions.

If we define $w_{0}=u_{0}+s_{0}$ we then obtain

$$
\begin{gathered}
\nabla^{2} w_{0}=0 \\
w_{0}(0, y, t)=u^{0}(y)+\frac{u^{0}(y)}{\lambda b\left(u^{0}(y)\right)}\left(1-e^{-\lambda b\left(u^{0}(y)\right) t}\right) \\
w_{0}(1, y, t)=u^{1}(y)+\frac{u^{1}(y)}{\lambda b\left(u^{1}(y)\right)}\left(1-e^{-\lambda b\left(u^{1}(y)\right) t}\right), \\
\left.\frac{\partial w_{0}}{\partial y}\right|_{y=0, l}=0 .
\end{gathered}
$$

Equation (4.9) is a Laplace problem with time-dependent boundary conditions with solution $w_{0}(x, y, t)$, which can be constructed using elementary separation of variables. Using the relation $s_{0}=w_{0}-u_{0}$ to eliminate $s_{0}$ from $(4.8 \mathrm{~b})$ yields

$$
\frac{\partial u_{0}}{\partial t}=\frac{\partial w_{0}}{\partial t}+\lambda b\left(u_{0}\right)\left(w_{0}-u_{0}\right)-u_{0}
$$

an inhomogeneous, nonlinear evolution equation for the concentration $u_{0}(x, y, t)$. We will now show how this equation can be written in a more suggestive form and then examine its behavior in the following section.

Recalling problem $(3.8 \mathrm{a}-\mathrm{c})$ for the time-independent solution $\bar{w}$ motivates rewriting boundary conditions (4.9b) using (3.7) and (3.8b) as

$$
\begin{aligned}
& w_{0}(0, y, t)=w^{0}(y)-\frac{u^{0}(y)}{\lambda b\left(u^{0}(y)\right)} e^{-\lambda b\left(u^{0}(y)\right) t} \\
& w_{0}(1, y, t)=w^{1}(y)-\frac{u^{1}(y)}{\lambda b\left(u^{1}(y)\right)} e^{-\lambda b\left(u^{1}(y)\right) t} .
\end{aligned}
$$


We can write $w_{0}$ as the sum of the time-independent solution and a transient term $\tilde{w}(x, y, t)$.

$$
w_{0}(x, y, t)=\bar{w}(x, y)+\tilde{w}(x, y, t)
$$

where $\tilde{w}(x, y, t)$ satisfies

$$
\begin{gathered}
\nabla^{2} \tilde{w}=0, \\
\tilde{w}(0, y, t)=-\frac{u^{0}(y)}{\lambda b\left(u^{0}(y)\right)} e^{-\lambda b\left(u^{0}(y)\right) t} \\
\tilde{w}(1, y, t)=-\frac{u^{1}(y)}{\lambda b\left(u^{1}(y)\right)} e^{-\lambda b\left(u^{1}(y)\right) t}, \\
\left.\frac{\partial \tilde{w}}{\partial y}\right|_{y=0, l}=0 .
\end{gathered}
$$

Using (4.12) and (3.7), (4.10) becomes

$$
\frac{\partial u_{0}}{\partial t}=\lambda b\left(u_{0}\right)\left[\left(\tilde{w}+\frac{1}{\lambda b\left(u_{0}\right)} \frac{\partial \tilde{w}}{\partial t}\right)+\left(\bar{w}(x, y)-W\left(u_{0}\right)\right)\right] .
$$

The solution of (4.14) will approach a steady state if the right-hand side of the equation vanishes as $t \rightarrow \infty$. Since $\tilde{w}$ vanishes exponentially everywhere as $t \rightarrow$ $\infty$, solutions will approach a steady state only if $\bar{w}(x, y)-W\left(u_{0}\right) \rightarrow 0$ everywhere. Recalling (3.9), this condition can be written as

$$
W\left(u_{0}(x, y, t)\right) \rightarrow W(\bar{u}(x, y)) \quad \text { as } t \rightarrow \infty .
$$

Recall that $\bar{u}(x, y)$ is a multivalued function, and $u_{0}(x, y, t)$ is necessarily single-valued. Numerical integration of (4.14) shows that $u_{0}$ develops a shock-like structure. In regions of the plane where $\bar{u}(x, y)$ is single-valued $u_{0}$ must converge to that value. Where $\bar{u}$ is multivalued it appears that $u_{0}$ has a choice about which value it will converge to. In the next section we will demonstrate that as $t \rightarrow \infty$ the solution approaches the discontinuous form

$$
u_{0}(x, y, t \rightarrow \infty) \rightarrow\left\{\begin{array}{ll}
\bar{u}_{-}(x, y) & \text { on left of shock } \\
\bar{u}_{*}(x, y) & \text { on the shock } \\
\bar{u}_{+}(x, y) & \text { on right of shock }
\end{array},\right.
$$

where $\bar{u}_{-}, \bar{u}_{+}$, and $\bar{u}_{*}$ are the three single-valued solution branches of (3.9) (see Fig. 5).

5. Shock formation. In this section we will examine how evolution equations of the form (4.14) form shocks. This analysis readily applies to multidimensional problems, as will be discussed, but for ease of presentation, we will show the argument for the one-dimensional version of the problem on $0<x<1$,

$$
\begin{gathered}
\epsilon u_{t}=u_{x x}+s_{x x}, \\
s_{t}+\lambda b(u) s=u, \\
u(0, t)=r, \quad u(1, t)=1,
\end{gathered}
$$




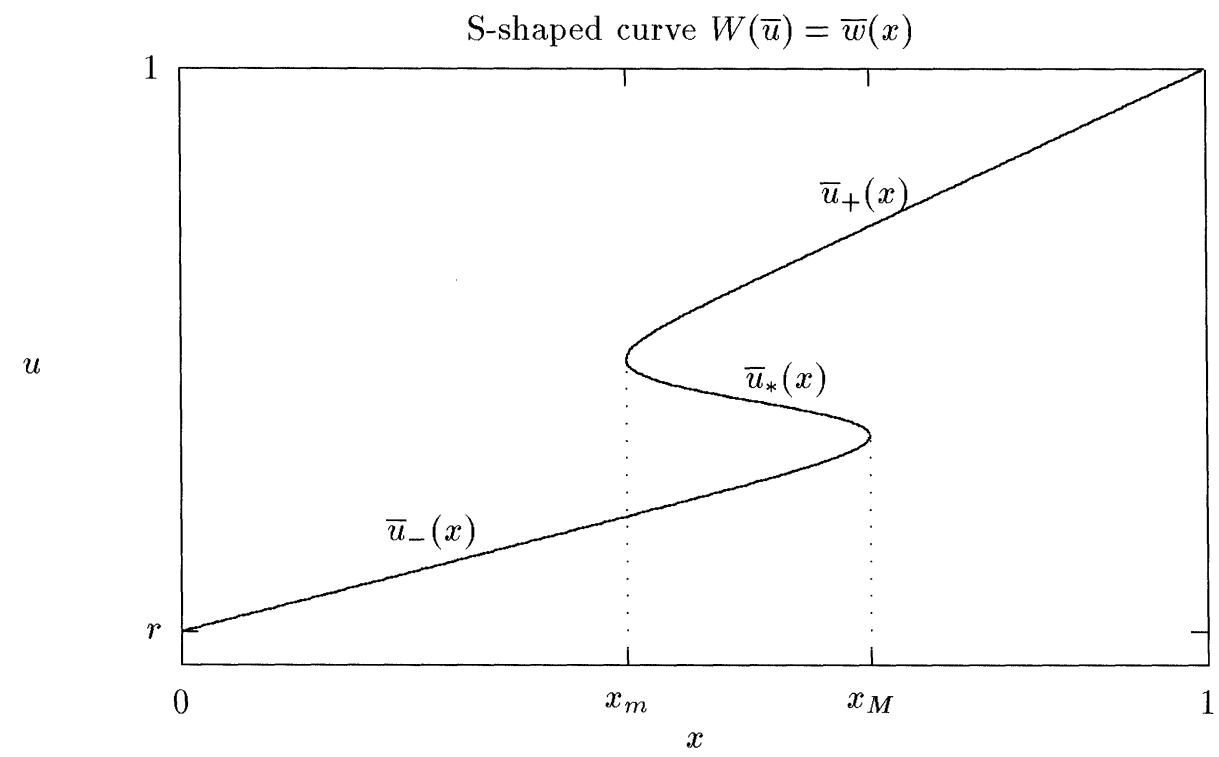

FIG. 5. Branches of the multivalued steady-state solution.

where $r<1$ is a constant, and

$$
u(x, 0)=0, \quad s(x, 0)=0 .
$$

Following the method of matched asymptotic expansions used in the earlier sections we find that the long-time nonlinear evolution equation for $u_{0}$ is

$$
\frac{\partial u_{0}}{\partial t}=\lambda b\left(u_{0}\right)\left[\left(\tilde{w}+\frac{1}{\lambda b\left(u_{0}\right)} \frac{\partial \tilde{w}}{\partial t}\right)+\left(\bar{w}(x)-W\left(u_{0}\right)\right)\right],
$$

with initial condition

$$
u_{0}(x, 0)=\underline{u_{0}}(x)=r(1-x)+x,
$$

where

$$
\tilde{w}(x, t)=-\frac{r}{\lambda b(r)} e^{-\lambda b(r) t}(1-x)-\frac{1}{\lambda b(1)} e^{-\lambda b(1) t} x,
$$

and the time-independent solution is

$$
\bar{w}(x)=W(r)(1-x)+W(1) x .
$$

To point out some of the interesting features of (5.2) we contrast it with an analogous evolution equation derived from a traditional reaction-diffusion system. Consider the following reaction-diffusion system, typical of systems examined by Fife [17] and others,

$$
\begin{gathered}
u_{t}=\epsilon^{2} u_{x x}+\lambda b(u)(\Gamma-(u+s)), \\
\epsilon s_{t}=\epsilon^{2} s_{x x}+s-\frac{u}{\lambda b(u)},
\end{gathered}
$$

where $\Gamma$ is a constant and $\lambda, b(u)$ are the same as were used in the previous sections. 
Using asymptotic expansions, we derive the leading-order evolution equation for the long-time asymptotic behavior of $u$ as

$$
\frac{\partial u_{0}}{\partial t}=\lambda b\left(u_{0}\right)\left(\Gamma-W\left(u_{0}\right)\right)
$$

where $W$ was defined in (3.7). To observe the formation of shocks in the system it is essential that $u=W^{-1}(\Gamma)$ is multivalued. Typically $W^{-1}$ is taken to be a triplevalued function (the well-known "S-shaped curve," see Fig. 6) that yields two stable equilibrium states over a parameter range $\Gamma \in\left[\Gamma_{m}, \Gamma_{M}\right]$. Note that this equation is translation invariant in space.

If we replace $\Gamma$ by the function $\bar{w}(x)$ then $(5.7)$ can be interpreted as resulting from a reaction-diffusion system in an inhomogeneous medium [18], and the corresponding leading-order evolution equation is

$$
\frac{\partial u_{0}}{\partial t}=\lambda b\left(u_{0}\right)\left(\bar{w}(x)-W\left(u_{0}\right)\right)
$$

This equation contains explicit spatial dependence, so it is no longer translation invariant. Therefore we do not expect to find that traveling wave front solutions exist for (5.8). In fact, we will find that (5.2) forms fixed-shock solutions.

Using $\bar{w}(x)$ given by (5.5) with (3.9), we can write an implicit representation of the time-independent solution

$$
x(\bar{u})=\frac{W(r)-W(\bar{u})}{W(r)-W(1)} .
$$

Define the "stable" branches $\bar{u}_{-}(x), \bar{u}_{+}(x)$ and the "unstable" branch $\bar{u}_{*}(x)$ of $(5.9)$ as indicated in Fig. 5. (The terms "stable" and "unstable" will be made rigorous later

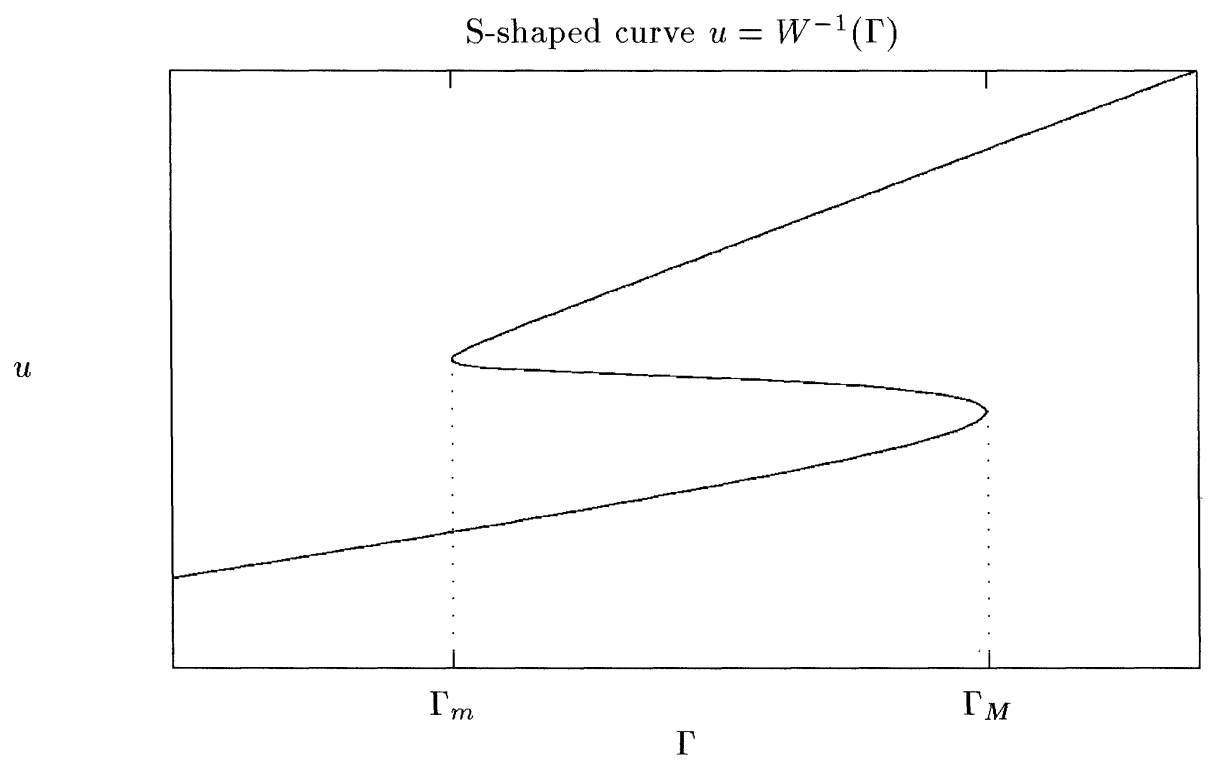

FIG. 6. The "S-shaped curve" given by $W^{-1}(\Gamma)$. 
in this section.) If we consider general initial conditions $u=\underline{u}(x)$ at $t=0$ for (5.8) we find that there are only two cases. If $\underline{u}(x)$ intersects the unstable branch $\bar{u}_{*}(x)$ then a shock will form at the intersection point $x_{s}$ such that $\underline{u}\left(x_{s}\right)=\bar{u}_{*}\left(x_{s}\right)$ (see Fig. 7). If $\underline{u}(x)$ does not intersect $\bar{u}_{*}(x)$ then a shock can only form at a turning point $x_{m}$ or $x_{M}$ (see Fig. 8).

As $t \rightarrow \infty, \tilde{w}$ vanishes exponentially quickly so we expect the steady-state Sshaped curve for (5.2) to be identical with that for (5.8). The nullcline for (5.2)

$u$

Shock Formation at $x_{s}$

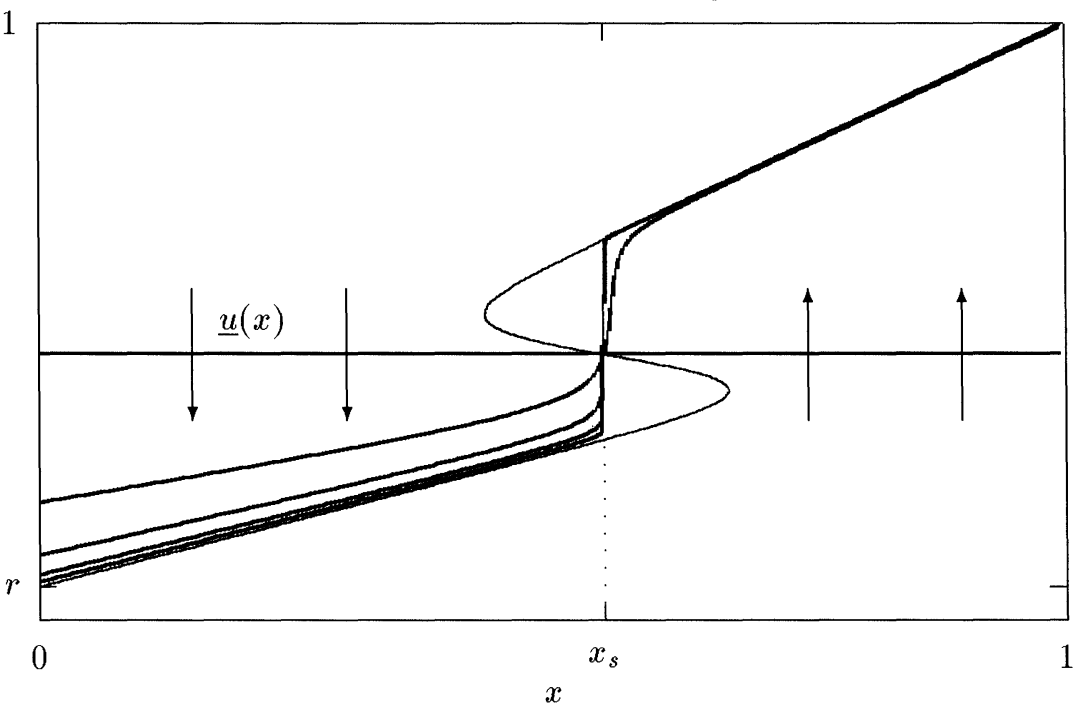

FIG. 7. Shock formation in the multivalued region.

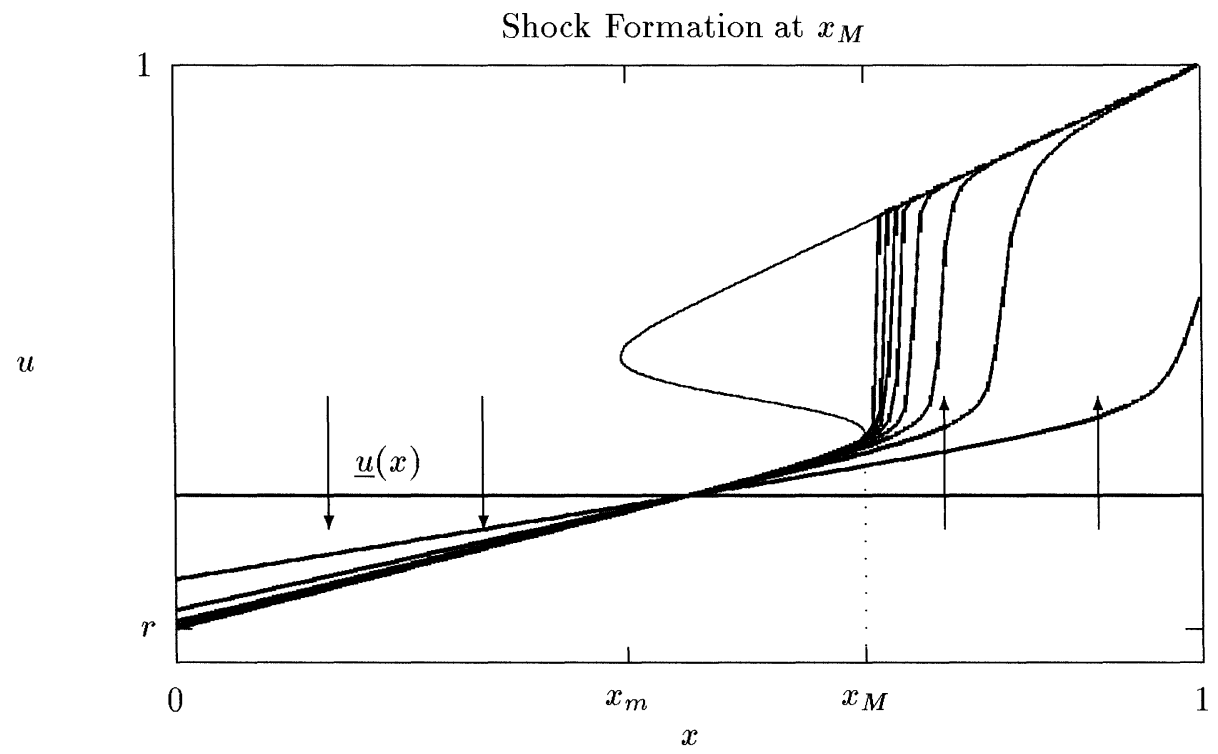

FIG. 8. Shock formation at a turning point. 
is

$$
x(u, t)=\frac{W(r)-W(u)+G(u, t, r)}{W(r)-W(1)+G(u, t, r)-G(u, t, 1)},
$$

where

$$
G(u, t, k)=k e^{-\lambda b(k) t}\left(\frac{1}{\lambda b(u)}-\frac{1}{\lambda b(k)}\right)
$$

which does converge to (5.9) as $t \rightarrow \infty$, therefore steady-state shocks can only exist in the same domain $x \in\left[x_{m}, x_{M}\right]$ where $\bar{u}$ is triple-valued. However, as described above, for (5.8) the shock position is determined by the initial condition at $t=0$. For (5.2) the inhomogeneous term makes determining the shock position a more delicate question since the analysis done for (5.8) does not hold (see Fig. 9). Equation (5.10) does not yield any information about shock position; what we wish to obtain is an "effective S-shaped curve" for (5.2)-a curve that determines the position of the steady-state shock for a given initial condition in our evolution equation.

We will explain the formation of the shock in $(5.1 \mathrm{a}-\mathrm{d})$ using a phase plane analysis. To recast (5.2) as an autonomous phase plane system we use the change of variables (and replace occurrences of $u_{0}$ by $u$ )

$$
v=e^{-\lambda b(r) t}, \quad \alpha=\frac{b(1)}{b(r)},
$$

and

$$
\tilde{w}(v ; x)=-\frac{r}{\lambda b(r)} v(1-x)-\frac{1}{\lambda b(1)} v^{\alpha} x
$$

Shock Formation with Time-Dependent Forcing

$u$

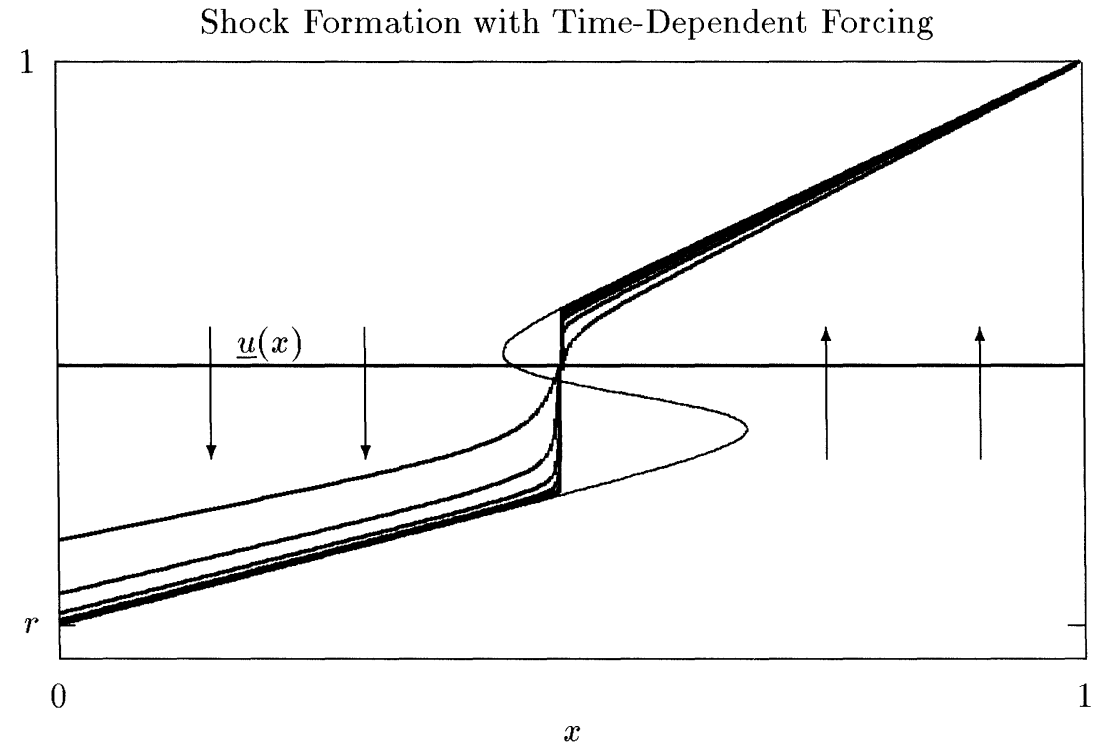

FIG. 9. Shock formation with time-dependent forcing. 
Then

$$
\frac{d u}{d t}=F(u, v ; x) \equiv \lambda b(u)\left[\left(\tilde{w}+\frac{1}{\lambda b(u)} \frac{\partial \tilde{w}}{\partial t}\right)+(\bar{w}(x)-W(u))\right]
$$

$$
\frac{d v}{d t}=-\lambda b(r) v
$$

Equations $(5.13 \mathrm{a}, \mathrm{b})$ are a phase plane system with $x$ as a parameter. When $W^{-1}$ is triple-valued, this system has three equilibrium points at $v=0$ and $\bar{u}$ corresponding to the three solutions of $\bar{w}(x)=W(\bar{u})$. Linear stability analysis yields the exponential growth rates, $\mu_{1,2}$, at the fixed points

$$
\mu_{1}=\left.\frac{\partial F}{\partial u}\right|_{\bar{u}, v=0}, \quad \mu_{2}=-\lambda b(r)<0 .
$$

In this phase plane representation, the stable branches of $W^{-1}$ correspond to stable nodes and the unstable branch corresponds to a hyperbolic saddle point (see Fig. 10).

Except for points on the stable manifold of the saddle point (the separatrix), all points are on trajectories that will approach one of the two stable nodes as $t \rightarrow \infty$ $(v \rightarrow 0)$. The separatrix is the boundary between the basins of attraction for the two stable nodes. Points on the separatrix will approach the saddle as $t \rightarrow \infty$. On the line $v=0$ are heteroclinic connections from the saddle to the stable nodes; this is the unstable manifold of the saddle. The interpretation of the phase plane is that if the initial condition $u$ at $t=0(v=1)$ is on the separatrix for parameter $x_{s}$, then as $t \rightarrow \infty$, $u$ will approach the unstable branch of $\bar{u}=W^{-1}(\bar{w}(x))$ and will form a shock at $x_{s}$ connecting it to the stable branches at $v=0$ (infinite time)(see Fig. 10). Interestingly, this can be interpreted as the statement that system $(5.1 \mathrm{a}-\mathrm{d})$ only forms shocks after infinite time, a fact that was rigorously proved by Amann [1], [2].

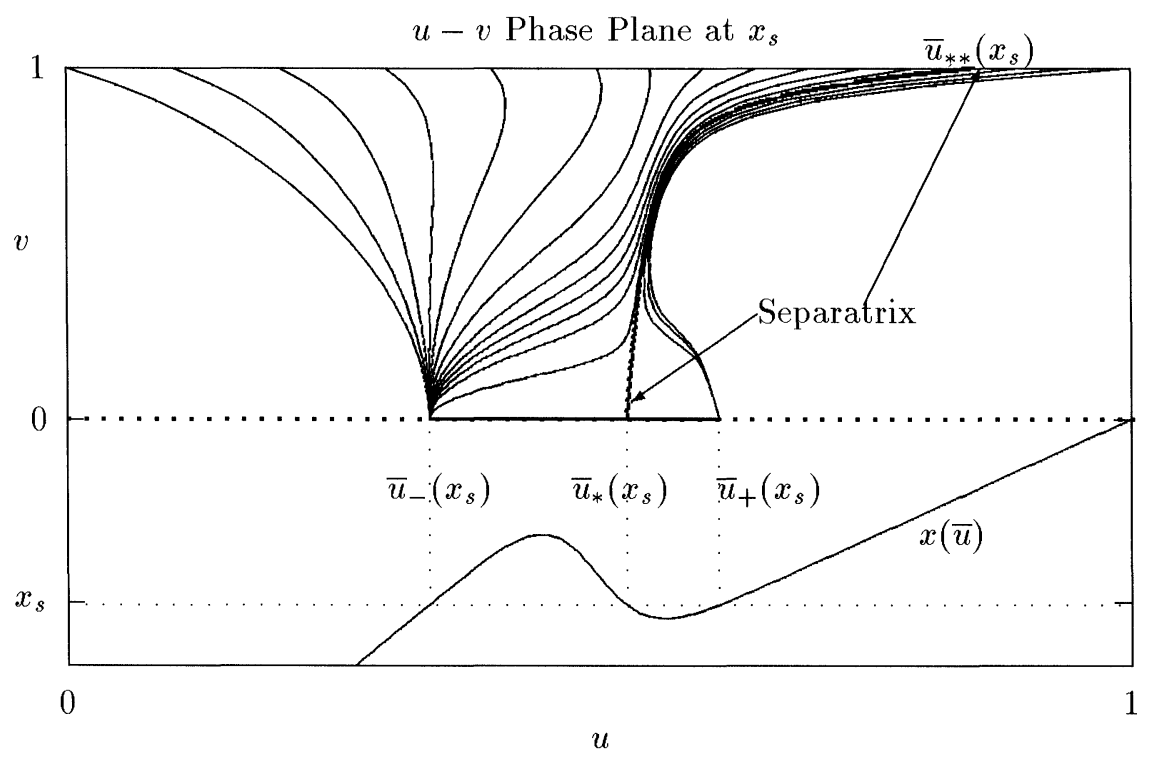

FIG. 10. The phase plane. 
By putting $(5.13 \mathrm{a}, \mathrm{b})$ in the form

$$
\frac{d u}{d v}=-\frac{F(u, v ; x)}{\lambda b(r) v}, \quad u(v=0 ; x)=\bar{u}_{*}(x),
$$

determination of condition required for shock formation is reduced to a problem of numerical integration on a finite domain to determine the value $\bar{u}_{* *}(x) \equiv u(v=1 ; x)$ for all $x \in\left[x_{m}, x_{M}\right]$. By comparison, for $(5.8), \bar{u}_{* *}(x)=\bar{u}_{*}(x)$ since the S-shaped curve is independent of time (the $u-v$ phase plane is translation invariant in $v$ ). The right-hand side of (5.15) is an indefinite form at the saddle point $u=\bar{u}_{*}(x), v=0$; but an application of l'Hôpital's rule yields an applicable initial condition as $v \rightarrow 0$

$$
\left.\frac{d u}{d v}\right|_{\bar{u}_{*}, v=0}=-\frac{\frac{\partial F}{\partial v}}{\lambda b(r)+\frac{\partial F}{\partial u}}, \quad u(v=\Delta v) \sim \bar{u}_{*}(x)+\frac{d u}{d v} \Delta v .
$$

This procedure yields the curve $\bar{u}_{* *}(x)$, the unstable branch of the "effective S-shaped curve." Again, its significance is that the steady-state shock will form at the position $x_{s}$ where the initial condition intersects $\bar{u}_{* *}, \underline{u}\left(x_{s}\right)=\bar{u}_{* *}\left(x_{s}\right)$. From Fig. 11, we see that $\bar{u}_{* *}(x)$ for $u \in[0,1]$ might not cover the whole unstable branch $\bar{u}_{*}(x), x \in$ $\left[x_{m}, x_{M}\right]$ and therefore one of the effects of the inhomogeneous time-dependent forcing is to reduce the domain where shocks can form.

The extension to two-dimensional problems is straightforward; we solve

$$
\frac{d u}{d v}=-\frac{F(u, v ; x, y)}{\lambda b\left(u^{0}(y)\right) v} \quad u(v=0 ; x, y)=\bar{u}_{*}(x, y)
$$

for all $(x, y)$ in the region of the plane where $\bar{u}(x, y)$ is triple-valued. In this case we will find that generally the intersection $\underline{u}\left(x_{s}, y_{s}\right)=\bar{u}_{* *}\left(x_{s}, y_{s}\right)$ will be a curved shock in the plane. Recall that for our problem $(3.3 \mathrm{a}-\mathrm{e})$ the initial condition for the outer evolution equation is given by $\underline{U_{0}}$ from problem $(4.6 \mathrm{a}-\mathrm{c})$. We plot a few more

$u$

Shock Position Determination

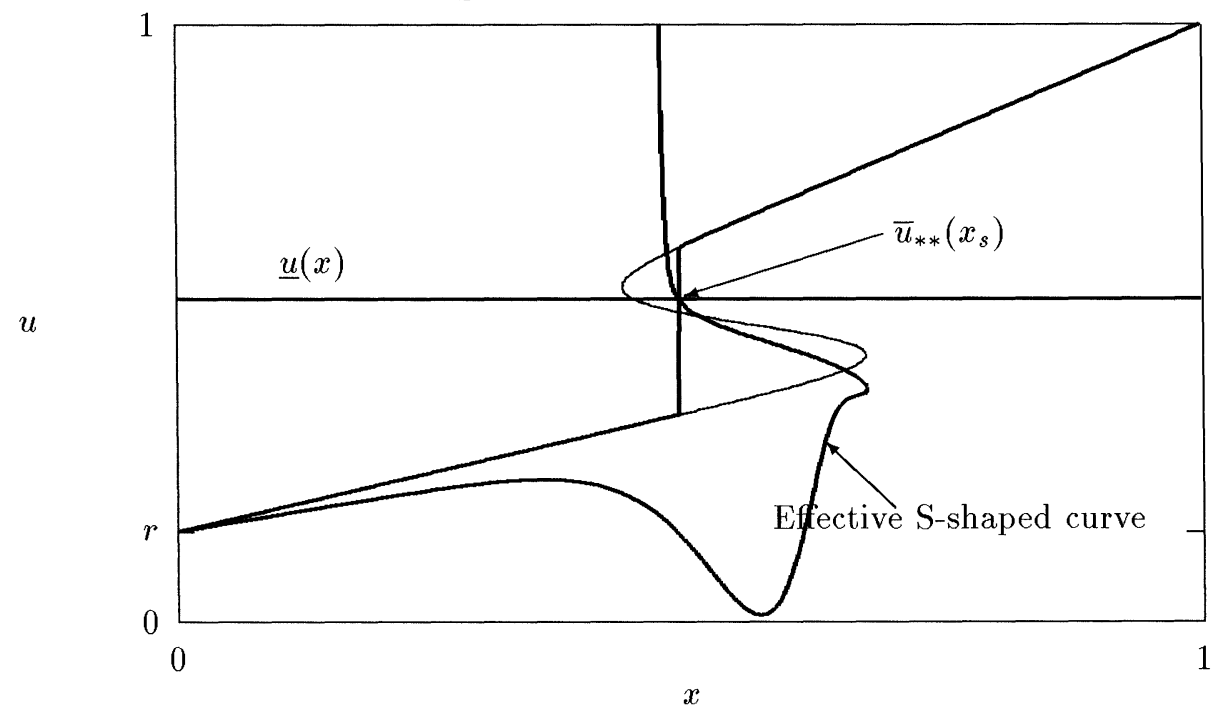

FIG. 11. The effective "S-shaped curve" for shock formation with time-dependent forcing. 


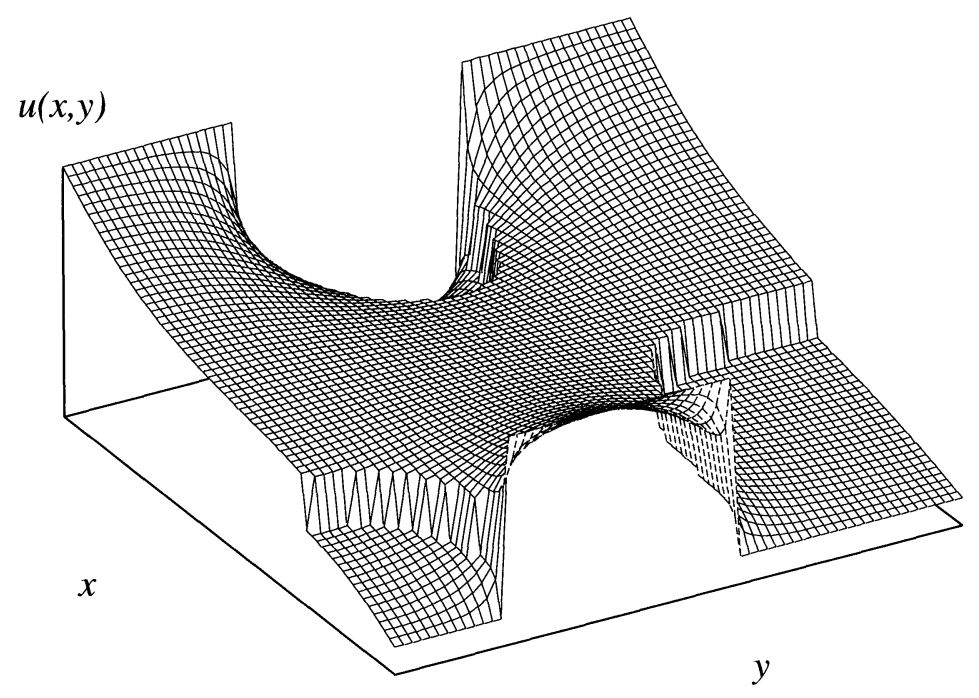

FIG. 12. Shock formation in a two-dimensional system.

dramatic examples of shock formation generated by specifying various boundary data $u^{0}(y), u^{1}(y)$ (see Figs. 12, 13).

6. Mass-uptake characteristics. As described earlier, in polymer-penetrant diffusion experiments observations typically include mass-uptake calculations, measurements of the amount of penetrant absorbed in the polymer film at each instance of time

$$
M(t)=\int_{0}^{1} u(x, t) d x
$$

In the results of Vrentas, Duda, and Hou [22], the mass-uptake curve initially shows a rapid increase, obtains a maximum value, slowly decreases, and then adjusts to a steady-state value; this is called sorption overshoot. We examine how this behavior is manifested in our model (again to simplify the argument, the analysis will be presented for the one-dimensional problem).

The observed rapid initial increase and gradual decrease behaviors of $M(t)$ can be readily explained in terms of the two asymptotic regimes used for the model. For a short range of initial times of order $\epsilon$ the absorption of the penetrant is governed by the classical heat equation (4.5) (Fickian diffusion). The analysis of the mass-uptake for this problem is well known [5], [6] and yields the rapid rise on the fast initial time scale $\tilde{t}$ until the steady state $(4.6 \mathrm{a}-\mathrm{c}),(5.4)$ is reached. For large finite times the behavior of the system is dominated by the interaction of the viscoelastic relaxation of the polymer with the fast diffusive equilibration of the penetrant (4.14), (5.2). The steady state, $M_{\infty}=M(t \rightarrow \infty)$, is determined by the position of the shock (as given 


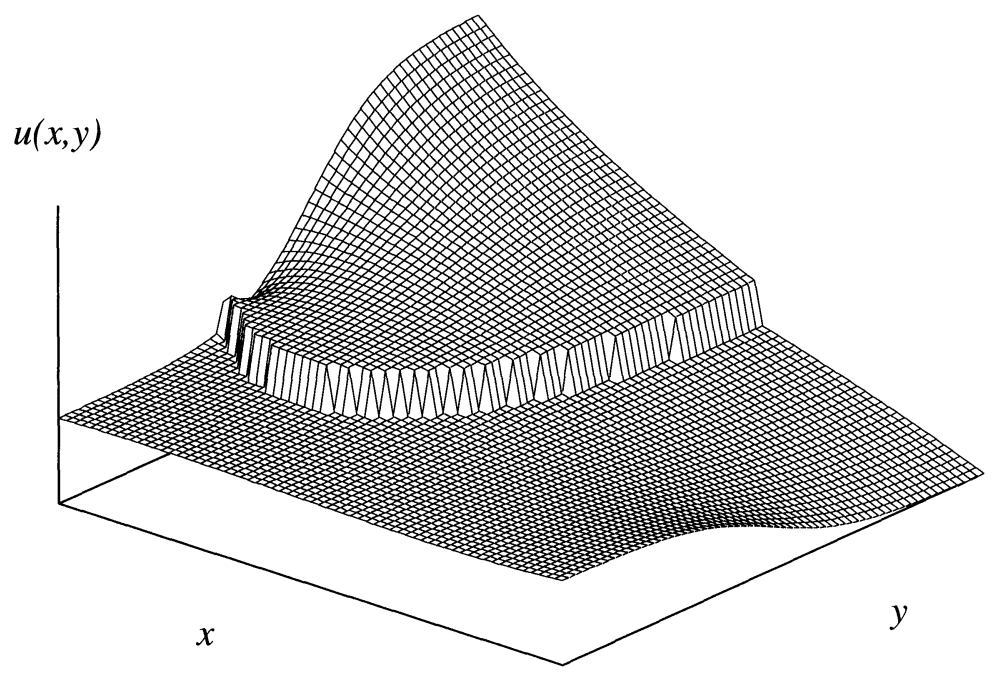

FIG. 13. Shock formation in a two-dimensional system.

from the results of the previous section)

$$
M_{\infty}=\int_{0}^{x_{s}} \bar{u}_{-}(x) d x+\int_{x_{s}}^{1} \bar{u}_{+}(x) d x .
$$

To understand the behavior of the outer solution for finite time it is convenient to assume an approximate form for the solution away from the shock and determine how rapidly it converges to the steady state. The ansatz and consistent approximations used for the following analysis are given by

$$
u(x, t) \sim\left\{\begin{array}{ll}
r(1-x)+k_{0}(t) x & x \ll x_{s} \\
k_{1}(t)(1-x)+x & x \gg x_{s}
\end{array},\right.
$$

$$
\text { away from } x_{s} \quad\left|u-u_{r g}\right| \gg O(\delta) \text {, }
$$

$$
\begin{array}{lll}
x \ll x_{s} & u \ll u_{r g} & b(u) \sim \omega, \\
x \gg x_{s} & u \gg u_{r g} & b(u) \sim 1 .
\end{array}
$$

Observe that if $k_{0}(0)=1$ and $k_{1}(0)=r$, then the ansatz satisfies the initial condition (5.4) and the boundary conditions (5.1c). Combining (6.3), (6.4) with (5.2), (5.5) yields

$$
\frac{d k_{0}}{d t} \sim-(1+\omega \lambda) k_{0}(t)+\omega(1+\lambda)+(1-\omega) e^{-\lambda t}
$$

$$
\frac{d k_{1}}{d t} \sim-(1+\lambda) k_{1}(t)+r(\lambda+1 / \omega)+r(1-1 / \omega) e^{-\lambda \omega t}
$$




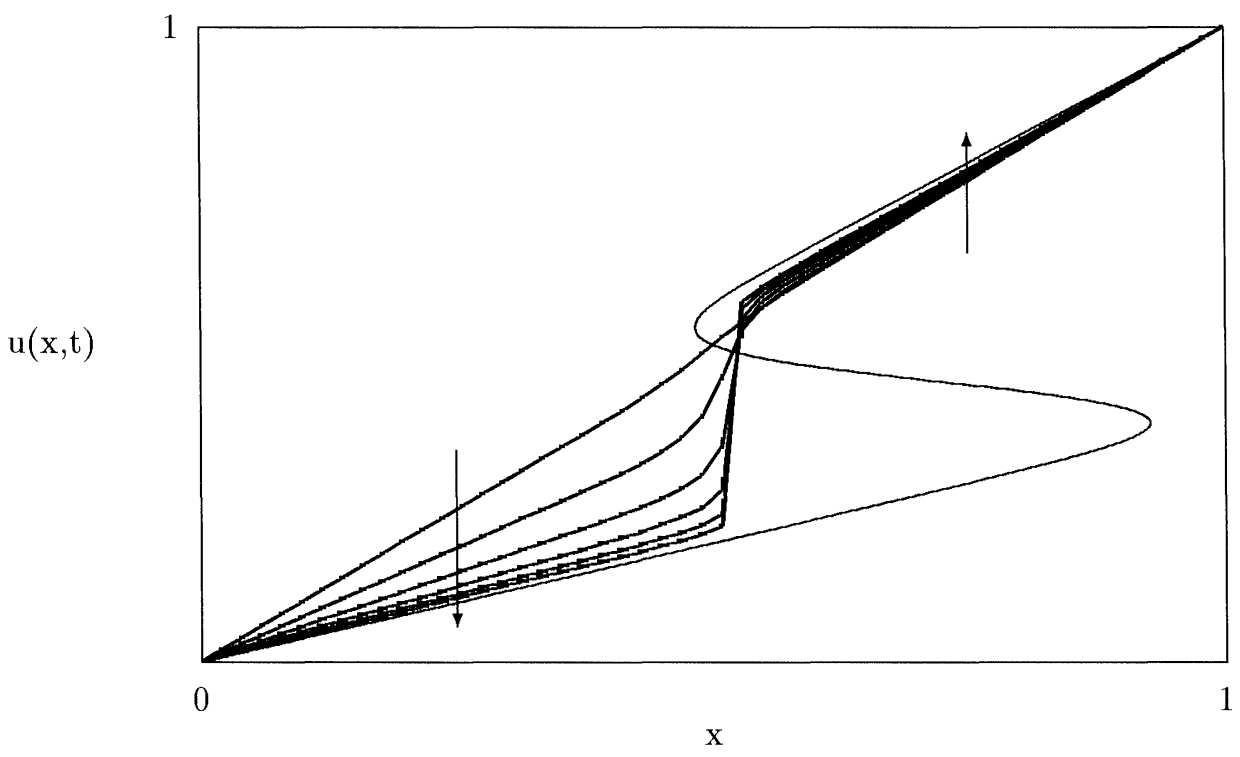

FIG. 14. Long-time evolution of the concentration profile.

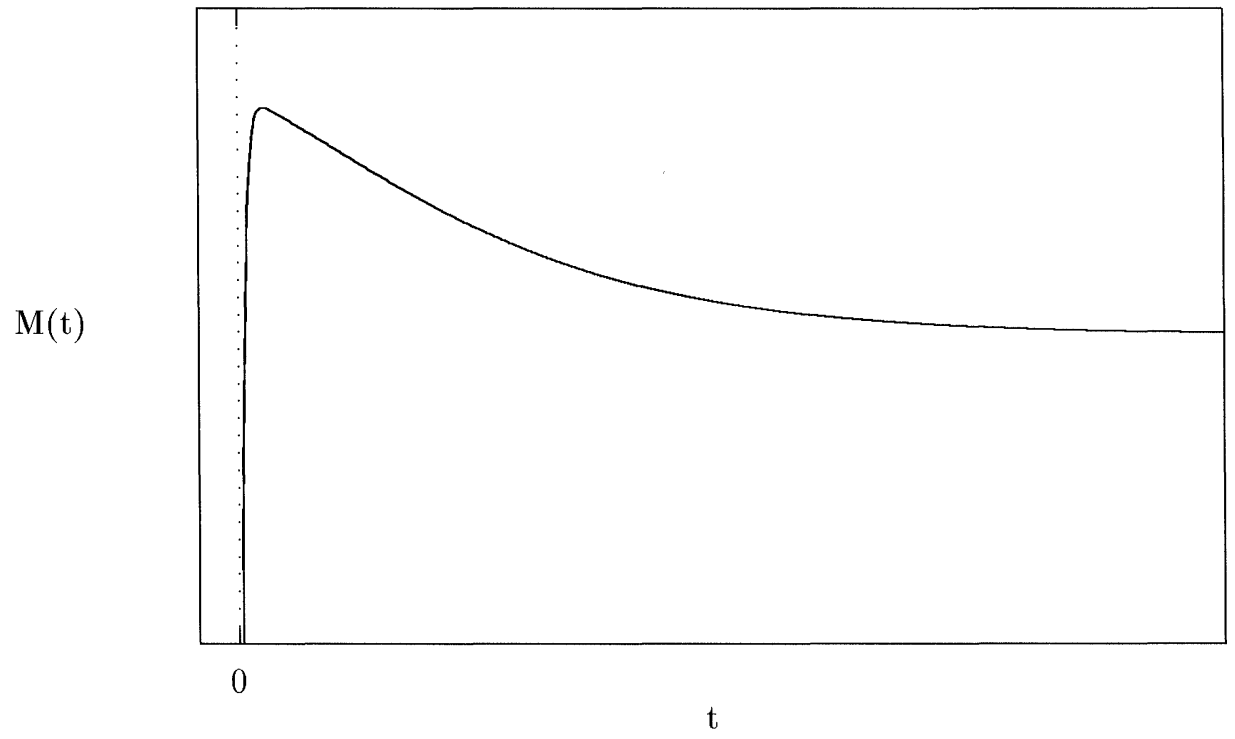

FIG. 15. A mass-uptake curve showing overshoot behavior.

Clearly $k_{0}(t)$ will have exponential decay rates $1+\omega \lambda$ and $\lambda$, and $k_{1}(t)$ has exponential decay rates $\omega \lambda$ and $1+\lambda$. From Fig. 14, time evolution of $k_{1}(t)$ tends to increase $M(t)$ while evolution of $k_{0}(t)$ tends to decrease $M(t)$. Therefore, depending on the values of $\lambda, \omega$, and $r$, we might observe $M(t)$ to have a local maximum or minimum then decay to a steady state, or simply monotonically go to a steady state depending on whether the contributions from $k_{0}(t)$ and $k_{1}(t)$ compete or one dominates. Therefore the Cohen-White model yields several possible classes of sorption behavior with overshoot being possible in a broad parameter range (see Fig. 15). 


\section{REFERENCES}

[1] H. AmANn, Nonhomogeneous linear and quasilinear elliptic and parabolic boundary value problems, in Function Spaces, Differential Operators and Nonlinear Analysis, H. Triebel and H. J. Schmeiszer eds., Teubner, Leipzig, 1993.

[2] - Highly degenerate quasilinear parabolic systems, Scuola Normale Superiore, 43 (1991), pp. $135-166$.

[3] D. S. Cohen And A. B. White JR., Sharp fronts due to diffusion and stress at the glass transition in polymers, J. Polymer Sci. B: Polymer Physics, 27 (1989), pp. 1731-1747.

[4] - Sharp fronts due to diffusion and viscoelastic relaxation in polymers, SIAM J. Appl. Math., 51 (1991), pp. 472-483.

[5] J. Crank, The Mathematics of Diffusion, 2nd ed., Oxford University Press, Oxford, 1975.

[6] J. Crank And G. S. PARK, Diffusion in Polymers, Academic Press, London, 1968.

[7] C. J. Durning, Differential sorption in viscoelastic-fluids, J. Polymer Sci. B: Polymer Physics, 23 (1985), pp. 1831-1855.

[8] C. K. HAYES AND D. S. CoHEN, The evolution of steep fronts in non-Fickian polymer-penetrant systems, J. Polymer Sci. B: Polymer Physics, 30 (1992), pp. 145-161.

[9] - Diffusion and Stress Driven Flow in Polymers, Ph.D. thesis, California Institute of Technology, 1990.

[10] R. W. Cox AND D. S. Cohen, A mathematical model for stress-driven diffusion in polymers, J. Polymer Sci. B: Polymer Physics, 27 (1989), pp. 589-602.

[11] R. W. Cox, A model for stress-driven diffusion in polymers, Ph.D. thesis, California Institute of Technology, 1988.

[12] - Shocks in a model for stress-driven diffusion, SIAM J. Appl. Math., 50 (1990), pp. 12841299.

[13] H. L. FRISCH, Sorption and transport in glassy polymers-a review, Polymer Engr. and Sci., 20 (1980), pp. 2-13.

[14] T. L. SMith AND R. E. AdAM, Effect of tensile deformations on gas transport in glassy polymer films, Polymer, 22 (1981), pp. 299-304.

[15] D. A. EDwARds, Heavily stressed polymer-penetrant system exhibiting two fronts, Ph.D. thesis, California Institute of Technology, 1994.

[16] C. J. Durning, D. S. Cohen, And D. A. Edwards, Perturbation analysis of Thomas' and Windle's model of Case II diffusion, to appear.

[17] P. C. FIFE, Singular perturbation and wave front techniques in reaction diffusion problems, in SIAM-AMS Proceedings, Symposium on Asymptotic Methods and Singular Perturbations, Vol. 10, R. E. O'Malley, Jr., ed., American Mathematical Society, Providence, RI, 1976, pp. 23-49.

[18] — Dynamics of Internal Layers and Diffusive Interfaces, Society for Industrial and Applied Mathematics, Philadelphia, 1988.

[19] W. FlugGe, Viscoelasticity, Springer-Verlag, New York, Berlin, 1975.

[20] W. G. KNAuss AND V. H. KenneR, On the hygrothermomechanical characterization of polyvinyl acetate, J. Appl. Physics, 51 (1980), pp. 5131-5136.

[21] N. L. Thomas and A. H. Windle, A theory of case II diffusion, Polymer, 23 (1982), pp. 529542 .

[22] J. S. VRentas, J. L. Duda, and A. C. Hou, Anomalous sorption in poly(ethyl methacrylate), J. Appl. Polymer Sci., 29 (1984), pp. 399-406. 